\title{
A Retrospective 2-Year Follow-up of Late Complications Treated Surgically and Endoscopically After Laparoscopic Roux-en-Y Gastric Bypass (LRYGB) and Laparoscopic Sleeve Gastrectomy (LSG) for Morbid Obesity
}

\author{
Javanainen, Mervi
}

2018-04

\begin{abstract}
Javanainen , M , Penttilä , A , Mustonen , H , Juuti , A , Scheinin , T \& Leivonen , M 2018 , ' A Retrospective 2-Year Follow-up of Late Complications Treated Surgically and Endoscopically After Laparoscopic Roux-en-Y Gastric Bypass (LRYGB) and Laparoscopic Sleeve Gastrectomy (LSG) for Morbid Obesity ' , Obesity Surgery , vol. 28 , no. 4 , pp. 1055-1062 . https://doi.org/10.1007/s11695-017-2967-0
\end{abstract}

http://hdl.handle.net/10138/301298

https://doi.org/10.1007/s11695-017-2967-0

publishedVersion

Downloaded from Helda, University of Helsinki institutional repository.

This is an electronic reprint of the original article.

This reprint may differ from the original in pagination and typographic detail.

Please cite the original version. 


\title{
A Retrospective 2-Year Follow-up of Late Complications Treated Surgically and Endoscopically After Laparoscopic Roux-en-Y Gastric Bypass (LRYGB) and Laparoscopic Sleeve Gastrectomy (LSG) for Morbid Obesity
}

\author{
Mervi Javanainen $^{1}$ • Anne Penttilä ${ }^{1}$ • Harri Mustonen ${ }^{2}$ Anne Juuti ${ }^{1} \cdot$ Tom Scheinin $^{3}$ • \\ Marja Leivonen ${ }^{4}$
}

Published online: 28 October 2017

(C) Springer Science+Business Media, LLC 2017

\begin{abstract}
Background The laparoscopic Roux-en-Y gastric bypass (LRYGB) has been the gold standard for bariatric surgery, but recently, the laparoscopic sleeve gastrectomy (LSG) has gained popularity. At present, limited data is available on the long-term complications of these two types of surgery. The aim of this retrospective study was to compare the 2-year data about late (more than 30 days after surgery) complications that were treated surgically or endoscopically after LRYGB and LSG operations in a large hospital area with a single patient database.
\end{abstract}

\section{Mervi Javanainen}

mervi.javanainen@hus.fi

Anne Penttilä

Anne.penttila@hus.fi

Harri Mustonen

harri.mustonen@helsinki.fi

Anne Juuti

Anne.juuti@hus.fi

Tom Scheinin

tom.scheinin@hus.fi

Marja Leivonen

Marja.Leivonen@epshp.fi

1 Meilahti University Hospital, Haartmaninkatu 4, 00290 Helsinki, Finland

2 Biomedicum Helsinki, Department of Surgery, Helsinki University Central Hospital, Haartmaninkatu 8, 00290 Helsinki, Finland

3 Jorvi Hospital, Turuntie 150, 02740 Espoo, Finland

4 Seinäjoki Central Hospital, Hanneksenrinne 7. 60220 Seinäjoki, Finland
Materials This was a retrospective, non-randomized, singlecenter study of 760 (545 LRYGB and 215 LSG) bariatric patients surgically treated between 2008 and 2013 in the Bariatric Surgery Unit of Helsinki University Central Hospital.

Methods The patients were followed for 2 years, and late complications (more than 30 days after surgery) that were surgically and/or endoscopically treated were registered. Weight loss and the risk factors for complications were also monitored.

Results The study found a difference between the LRYGB and LSG patients in a number of late complications treated by both intervention types: surgical intervention were required in $9.4 \%$ of LRYGB patients vs. 0.9 of LSG patients, and endoscopic intervention were required by $4.6 \%$ of LRYGB patients vs. $1.4 \%$ of LSG patients (both $p<0.05$ ). The risk of surgical complications was increased by better weight loss results in 12 months.

Conclusions LRYGB was found to be associated with a greater risk of late complications. If larger databases confirm these results, the trend toward LSG is justified.

Keywords Bariatric surgery $\cdot$ Late complications $\cdot$ LSG · LRYGB

\section{Introduction}

Obesity is a chronic disease said to result from a combination of environmental and genetic factors [1]. In the USA, it is the second most common cause of preventable deaths [2]. In 2013, the number of bariatric procedures worldwide was estimated at 468,609 and $95.7 \%$ were carried out laparoscopically. The most popular operations were 
Roux-en-Y gastric bypass (RYGB) (45\%), sleeve gastrectomy (SG) $(37 \%)$, and adjustable gastric banding (10\%). Between 2003 and 2013, there was a significant increase in the popularity of SG, from 0 to $37 \%$ [3].

The laparoscopic RYGB (LRYGB) has been the gold standard for bariatric surgery, but lately, the laparoscopic SG (LSG) has been gaining popularity [4]. The Cochrane Update review of bariatric surgery stated that LRYGB and LSG are comparable in terms of improvement of comorbidities and weight loss [5]. However, recent data suggest that LRYGB might have more early and late complications than LSG [6, 7], which could further change the relative popularity of these surgery types in the future.

Limited data are available on the number of late complications after LRYGB and LSG. Usually, patients with early postoperative complications are treated in specialized bariatric surgery centers, but patients who develop later complications may seek treatment at local surgical units, leading to gaps in the data.

The aim of this retrospective study was to compare the 2year data on late (more than 30 days after surgery) complications of LRYGB and LSG operations that were surgically or endoscopically treated in a large hospital area with a single patient database.

\section{Methods}

This was a retrospective, non-randomized study of 760 bariatric patients surgically treated between 2008 and 2013 at Peijas Hospital, in the Bariatric Surgery Unit of Helsinki University Central Hospital. The retrospective data included both primary and revisional bariatric surgery patients. There were originally 772 patients, of which 12 were excluded. These included 2 gastric bandings, 9 band removals, and 1 duodenal switch. Therefore, the study included only LRYGB and LSG patients.

Preoperatively, the patients had undergone medical, nutritional, and psychological screening. The patients had been selected using criteria similar to those recommended in the European Guidelines on Metabolic and Bariatric Surgery [8]. Preoperative upper stomach ultrasound and gastroscopy had been performed. If a Helicobacter pylori infection had been diagnosed, it was treated prior to the operation. Diagnosed gallstones had not been removed prior to the bariatric operation but rather, if symptomatic, were removed electively from 2 to 6 months after the bariatric surgery.

The selection of operation type was generally based on the surgeons' and patients' preferences. LSG was recommended for patients who were extremely obese or had extra risk factors, including severe intra-abdominal adhesions after previous abdominal operations, liver cirrhosis, chronic obstructive pulmonary disease, chronic renal failure, coagulopathy, and risk factors for malignancy in the stomach. However, in 2009-2010, Peijas Hospital took part in a multicenter study in which the type of operation was randomized between LRYGB and LSG [9].

The present study followed bariatric patients in the hospital district for 2 years and registered their late surgical complications. We also monitored weight loss. The first follow-up was at 1 month postoperatively and was conducted by the surgeon; thereafter, follow-ups were conducted by an endocrinologist at 3, 6, 12, and 24 months. In addition, the patients were requested to contact the hospital if they had problems that they thought were related to their bariatric surgery.

During the 2-year follow-up period, 10 patients died of causes unrelated to the bariatric surgery, 78 were followed in other hospital districts, and 3 refused the follow-ups. However, these patients were included in the study, because their problems related to obesity surgery were treated in the study hospital.

As a primary endpoint, the present study monitored the numbers of late surgical complications of LRYGB and LSG, including those treated with either surgical or endoscopic interventions. Potential risk factors, including weight loss, age, gender, and comorbidities, were analyzed. In addition, the time between the primary operation and the first intervention for complication was analyzed. Data were collected prospectively by the authors. The study was approved by the Finnish Ethical Committee.

\section{Operation Techniques}

Gastric Bypass Two 12-mm and three 5-mm dilating ports were used, three on the left side of the abdomen, one on the right side, and one under the xiphoideum to hold the liver retractor. A 20-ml gastric pouch was formed with three to five linear stapler firings (Endo-GIA; Medtronic; Minneapolis, $\mathrm{MN}$, USA). The first 100 gastro-jejunal anastomoses were formed using a circular stapler technique (Circular stapler; Orvil; Medtronic; Minneapolis, MN, USA) and, thereafter, using a linear stapler technique, with hand-sewing employed to close the stapler-device hole. The length of the biliopancreatic channel was between 60 and $80 \mathrm{~cm}$ and that of the alimentary channel $150 \mathrm{~cm}$. The loop was ante-colic. The jejuno-jejunal anastomosis was made with a linear stapler and hand-sewing to close the stapler-device hole. The biliary and alimentary channels were divided after the enteroanastomosis was formed. Meso-openings were closed using various methods and absorbable or non-absorbable suturing or metallic clips. The gastro-jejunal anastomosis was tested using $50 \mathrm{ml}$ of methylene blue dye.

Sleeve Gastrectomy Two 12-mm and one 5-mm dilating ports were employed on the left upper abdomen, one $15-\mathrm{mm}$ port on the right abdomen, and one $5-\mathrm{mm}$ port under the xiphoideum to hold the liver retractor. The sleeve was formed 
with the help of a 35-Fr boogie. The procedure was initiated by dividing the vessels along the greater curvature, from $6-\mathrm{cm}$ proximal from the pylorus up to the angle of Hiss, and the adhesions behind the stomach were cut. The resection was started from the antrum, with 45- and 60-mm green staplers (Endo-GIA; Medtronic; Minneapolis, MN, USA) and then with green/golden $60-\mathrm{mm}$ staplers along the boogie and up to the angle of Hiss, where a 1-cm corner of stomach was left. Reinforcements (Peri-Strip dry; Synovis; USA) were used on the stapler line, and the line was tested with $200 \mathrm{ml}$ of methylene blue dye.

Statistical analysis was performed using SPSS version 21 (IBM Corp.; New York, NY, USA) and SAS version 9.4 (SAS Systems; SAS Institute Inc.; Cary, NC, USA). Categorical variables are presented as numbers $(n)$ and percentages (\%) and continuous variables as means and standard deviations (SD) or medians and interquartile ranges (IQR). Continuous data were tested for normality using the Shapiro-Wilk test. Differences in continuous variables between groups were analyzed using the Kruskal-Wallis test or the Mann-Whitney $U$ test, and differences in binominal or categorical variables were analyzed using the Fisher's exact test or the Fisher-FreemanHalton test. Univariate and multivariate logistic regression were used to identify significant risk factors for complications. The Firth's penalized logistic regression was used in multivariate regression regarding complications without cholecystectomies. The excess weight loss percentage (EWL \%) was calculated as the ideal body weight to yield a body mass index (BMI) of $25 \mathrm{~kg} / \mathrm{m}^{2}$.

\section{Results}

The study included 760 patients, 545 LRYGB (71.7\%) and 215 LSG (28.3\%), of whom $68.9 \%$ were female. The LRYGB and LSG patient groups were similar regarding gender, comorbidities, and previous operations but not regarding age and pre-operative weight (Table 1).

There were 60 surgical interventions among the LRYGB patients. However, 6 patients had more than 1 surgical intervention or had 2 different diagnoses for a single operation; thus, the final number of LRYGB patients with operative complications was 51 , and the number of LSG patients was 2 . Four LSG patients were treated endoscopically, but one had two interventions, so the total number of patients with endoscopic complications was three. There was a significant difference in the number of patients in the LRYGB and LSG groups who were treated with both intervention types: $9.4 \%$ of LRYGB patients were treated surgically vs. $0.9 \%$ of LSG patients, and $4.6 \%$ of LRYGB patients were treated endoscopically vs. $1.4 \%$ of LSG patients (both $p<0.05$ ) (Table 2).

Table 3 presents the complications that were treated surgically without cholecystectomies. In this table, the total number
Table 1 Patient data and prior operations

\begin{tabular}{lllc}
\hline & LRYGB & LSG & $p$ \\
\hline Patients & 545 & 215 & \\
Sex, female & $381(69.9 \%)$ & $143(66.5 \%)$ & 0.39 \\
Age, median (IQR) & $47(23-65)$ & $49(24-67)$ & 0.023 \\
Pre-operative BMI (IQR) & $44.1(32.4-77.7)$ & $46.6(31.7-72.1)$ & $<0.001$ \\
Hypertension & $387(71 \%)$ & $145(67.4 \%)$ & 0.34 \\
Diabetes & $262(48.1 \%)$ & $107(49.8 \%)$ & 0.68 \\
Hypercholesterolemia & $183(33.6 \%)$ & $84(39.1 \%)$ & 0.17 \\
Sleep apnea & $160(29.4 \%)$ & $70(32.6 \%)$ & 0.43 \\
Prior cholecystectomy & $80(14.7 \%)$ & $38(17.7 \%)$ & 0.32 \\
Prior gastric banding & $6(1.1 \%)$ & $5(2.3 \%)$ & 0.31 \\
Prior abdominal surgery & $49(9 \%)$ & $10(4.7 \%)$ & 0.050 \\
\hline
\end{tabular}

$L R Y G B$ laparoscopic Roux-en-Y gastric bypass, $L S G$ laparoscopic sleeve gastrectomy, $I Q R$ interquartile range, $B M I$ body mass index

of patients treated was 31 in the LRYGB group and 0 in the LSG group. There was a significant difference $(p<0.001)$ between the groups.

The mean percentages of excess weight loss (EWL \%) at 1 and 2 years after the procedure differed between the two procedures, with those having undergone LRYGB losing more (Table 4).

The mean time until the first surgical intervention was 390 days for LRYGB and 388 days for LSG patients $(p=0.98)$, and for endoscopic interventions, it was 127 days for LRYGB and 209 days for LSG patients $(p=0.61)$ (Table 4). Figure 1 presents the timeline for the most common complications.

Multivariate logistic regression analysis (Table 5) found that for complications treated surgically, 12-month total weight loss was a risk factor and LSG was a preventative factor. Table 6 presents a logistic regression model without cholecystectomies, which shows similar findings. Neither age, gender, nor comorbidities affected the risk of complications in the multivariate model.

In the univariate regression analysis, EWL\% in 1 and 2 years were a risk factor for complications and LSG was a preventive factor. Prior cholecystectomy nor gastric banding did not affect the risk for surgical complications. However, all other previous abdominal operations, including ventral hernia, fundoplication, and cancer operations, were risk factors for complications. For unknown reasons, the univariate regression found hypertension to be a factor preventing complications. Reason for this cannot be explained (Table 7).

\section{Discussion}

The short-term complications of bariatric surgery have been widely recognized and analyzed. However, limited data is 
Table 2 Endoscopic and surgical interventions in LRYGB and LSG patients

\begin{tabular}{llll}
\hline Endoscopically treated (\%) & LRYGB(545) & LSG(215) & $p$ \\
\hline Marginal ulcer & $13(2.4 \%)$ & $1(0.5 \%)$ & 0.13 \\
Stenosis & $12(2.2 \%)$ & $1(0.5 \%)$ & 0.12 \\
Late fistulae & 0 & $1(0.5 \%)$ & 0.283 \\
Bezoar & 0 & $1(0.5 \%)$ & 0.283 \\
Total number of endoscopic complications & $25(4.6 \%)$ & $4(1.9 \%)$ & \\
Total number of patients with endoscopic complications & $25(4.6 \%)$ & $3(1.4 \%)$ & 0.034 \\
Surgically treated (\%) & & & \\
Internal hernia & $12(2.2 \%)$ & 0 & 0.024 \\
Incisional hernia & $3(0.6 \%)$ & 0 & 0.2 \\
SBO/strangulation & $7(1.3 \%)$ & 0 & 0.33 \\
Alimentary and biliary limb problems & $5(0.9 \%)$ & 0 & 0.58 \\
Abdominal pain NAS & $4(0.7 \%)$ & 0 & 0.58 \\
Perforation & $4(0.7 \%)$ & 0 & 1 \\
Invagination & $1(0.2 \%)$ & 0 & 1 \\
Fistula, laparoscopically treated & $1(0.2 \%)$ & 0 & \\
Cholecystectomy & & & 0.08 \\
Elective & $18(3.3 \%)$ & $2(0.9 \%)$ & 0.33 \\
Acute & $5(0.9 \%)$ & 0 & $2(0.9 \%)$ \\
Total number of surgical complications $(\%)$ & $60(11 \%)$ & $2(0.9 \%)$ & \\
Total number of patients with surgical complications $(\%)$ & $51(9.4 \%)$ & & \\
\hline
\end{tabular}

Three LRYGB patients had two surgical interventions or more than one diagnosis for a single operation and three patients had three surgical interventions or more than one diagnosis in one operation. One LSG patient had two endoscopic interventions

$L R Y G B$ laparoscopic Roux-en-Y gastric bypass, $L S G$ laparoscopic sleeve gastrectomy, $S B O$ small bowel obstruction, Abdominal pain NAS abdominal pain without abnormality seen in the operation available on the long-term complications of LRYGB and LSG operations.

Internal hernia $(\mathrm{IH})$ is one of the most common complications of LRYGB and can be fatal [10]. In a study of 594 LRYGB patients, the incidence of IH was $6.2 \%$, and $67.6 \%$ of IHs occurred at the Petersen's space and $24.3 \%$ at the jejuno-jejunostomy site [10]. In a recent meta-analysis of 31,320 bariatric patients, the incidence of $\mathrm{IH}$ varied from 0 to $16 \%$ [11], with the lowest incidence (1\%) found in groups in which an ante-colic approach was combined with the closure of all defects. Among the subjects in the present study, the incidence of IH was $2.2 \%$, and $50 \%$ of the IHs were at the jejuno-jejunostomy site and 50\% were at the Petersen's space.
As described, the technique at the hospital under study included an ante-colic approach and the closure of all meso-openings, using various methods. Because of the few instances of this complication (12 cases), it was not possible to make a useful comparison between the closing methods used for instances of IH. In addition, open internal space without IH was found in a re-operation six times in the Petersen's space and four times in the jejuno-jejunostomy site. These defects were closed during the re-operations. Of the open meso-openings, whether herniated or not, $22.7 \%$ were closed with absorbable sutures, $13.6 \%$ with clips, $22.9 \%$ were not closed, and the dispositions of $54.4 \%$ were not mentioned in the patient records.
Table 3 Surgical interventions in LRYGB and LSG patients without cholecystectomies

\begin{tabular}{llll}
\hline Surgically treated (\%) & LRYGB(545) & LSG(215) & p \\
\hline Internal hernia & $12(2.2 \%)$ & 0 & 0.024 \\
Incisional hernia & $3(0.6 \%)$ & 0 & 0.56 \\
Other surgical problems related to bariatric surgery & $22(4 \%)$ & 0 & 0.001 \\
Total number of surgical complications (\%) & $37(6.8 \%)$ & 0 & $<0.001$ \\
Total number of patients with surgical complications $(\%)$ & $31(5.7 \%)$ & 0 & \\
\hline
\end{tabular}

Six LRYGB patients had two surgical interventions or more than one diagnosis for a single operation $L R Y G B$ laparoscopic Roux-en-Y gastric bypass, $L S G$ laparoscopic sleeve gastrectomy 
Table 4 Excess weight loss percentage from the operation, missing patients, and time for the first intervention

\begin{tabular}{llll}
\hline & LRYGB(545) & LSG(215) & \\
\hline 1-year \%EWL (SD) & $49.7 \%(21.2)$ & $42.6 \%(22)$ & $<0.001$ \\
1-year missing weight data (\%) & $101(18.5 \%)$ & $36(16.7 \%)$ & \\
2 years \%EWL (SD) & $50.5 \%(22.7)$ & $45.5 \%(25.2)$ & 0.024 \\
2 years missing weight data (\%) & $228(41.8 \%)$ & $72(33.4 \%)$ & \\
Time for first surgically treated complication/day(SD) & $390(241)$ & $388(345)$ & 0.98 \\
Time for first endoscopically treated complication/day(SD) & $127(160)$ & $209(262)$ & 0.61 \\
\hline
\end{tabular}

The excess weight loss percentage (\%EWL) is calculated of ideal body weight to a BMI of $25 \mathrm{~kg} / \mathrm{m} 2$ $L R Y G B$ laparoscopic Roux-en-Y gastric bypass, $L S G$ laparoscopic sleeve gastrectomy, \%EWL excess weight loss percentage

Another study of 607 LRYGB patients found $25 \mathrm{IHs}$, of which 2 occurred in the immediate postoperative period and 23 occurred later, at a mean of 29 months after the operation [12]. The present study followed patients for only 24 months, so it is likely that some IHs occurred after that period. However, in the present study, the mean time for IH diagnosis was 15 months.

Incisional hernia of the anterior abdominal wall is a common complication after all abdominal surgeries and untreated, impairs quality of life [13]. Risk factors for incisional hernia after bariatric surgery have been shown to include an open surgical approach, prior abdominal surgery, malnutrition, and a BMI of more than $60 \mathrm{~kg} / \mathrm{m}^{2}$ [14]. Neither procedure type, complications, nor weight loss is associated with the occurrence of incisional hernias [15]. In a study of 2161 open or laparoscopic bariatric patients, $2.4 \%$ had an incisional hernia [14]. Another study of 1524 laparoscopic bariatric procedures found a $0.5 \%$ incidence of trocar port hernias and a $0.9 \%$ incidence of abdominal wall hernias [15]. In the present study, three patients $(0.6 \%)$ had incisional hernia after their operations.

Another common complication after LRYGB is small bowel obstruction (SBO), which can be caused by adhesion, internal hernia, incarcerated ventral hernia, or intussusception [6]. The present study found seven (1.3\%) SBO/strangulations and one $(0.2 \%)$ invagination after LRYGB.

Recurrent abdominal pain can occur after bariatric surgery, and diagnosis is important to exclude potentially lifethreatening abdominal catastrophes, such as IHs. Diagnoses are made using computed tomography, endoscopy, and upper gastrointestinal imaging [16]. However, sometimes the diagnosis remains unclear, and diagnostic laparoscopy is required
[16]. The use of diagnostic laparoscopy after bariatric surgery has not been well documented, although in a small retrospective study of 13 patients who had undergone diagnostic laparoscopic surgery after bariatric surgery, $2(15 \%)$ had negative findings [16]. The present study found $4(0.7 \%)$ operations with negative findings. Nonspecific abdominal pain after bariatric surgery can result from maladaptive eating behavior, increased food intolerance, constipation, or diarrhea, among other things [17].

With RYGB, marginal ulceration is seen in from 0.49 to $20 \%$ of patients [1]. Risk factors include smoking, diabetes, long gastric pouch, and Helicobacter pylori $[18,19]$. The ulceration is managed by treating the underlying problem, such as ceasing smoking and adding a proton-pump inhibitor for 36 months [1]. The present study found $13(2.4 \%)$ marginal ulcerations in LRYGB patients and $1(0.5 \%)(p=0.13)$ in an LSG patient. The mean time for ulcers was 21 weeks after the operation.

Stenosis commonly presents at a rate of $2-12 \%$ and at 4 8 weeks after an LRYGB operation, most commonly occurring at the gastro-enteric anastomoses but sometimes at the entero-enteric anastomosis [1]. Endoscopic balloon dilation of stricture/stenosis has proven to be an effective, safe therapeutic tool [20]. The number of dilatations needed varies but is usually from one to four [21]. The present study found 12 (2.2\%) cases of stenosis/stricture in LRYGB patients and 1 $(0.5 \%)$ case in an LSG patient $(p=0.12)$. The mean time for stenosis/stricture was 14.1 weeks after the operation.

It has been suggested that incomplete transection of the gastric pouch and gastric remnant can result in gastro-gastric fistula after LRYGB. Other common locations of enteric fistula are gastro-cutaneous and gastro-peritoneal [1]. Chronic

Fig. 1 Mean time in months for the most common surgically and endoscopically treated complications.

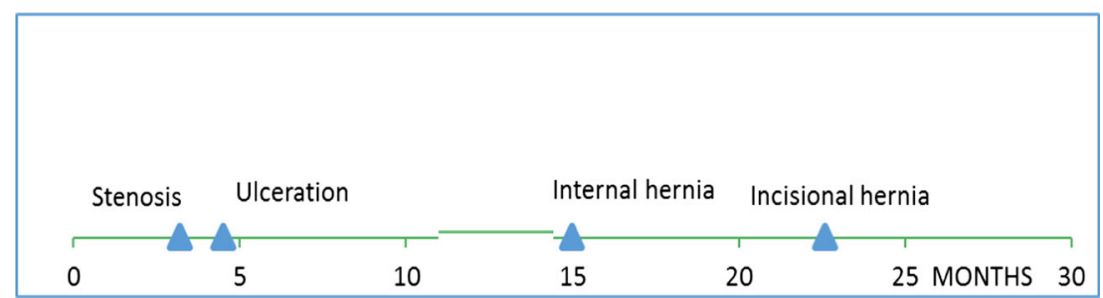


Table 5 Logistic multivariate regression analyze regarding age, comorbidities, sex, operation type, and weight loss regarding surgical complications

\begin{tabular}{lllll}
\hline & $p$ & ODDS & Lower (95\% CI) & Upper (95\% CI) \\
\hline Age $>$ 55 & 0.381 & 0.64 & 0.23 & 1.74 \\
Age 44-55 & 0.405 & 0.74 & 0.37 & 1.50 \\
Age $<45$ & & 1.0 & & \\
Sex (female) & 0.868 & 1.06 & 0.52 & 2.15 \\
Hypertension & 0.139 & 0.60 & 0.30 & 1.18 \\
Diabetes & 0.958 & 0.98 & 0.50 & 1.93 \\
LSG & 0.004 & 0.05 & 0.01 & 0.38 \\
Initial EWL\% 12 months & 0.025 & 1.02 & 1.00 & 1.03 \\
\hline
\end{tabular}

The excess weight loss percentage (\%EWL) is calculated of ideal body weight to a BMI of $25 \mathrm{~kg} / \mathrm{m} 2$ $L S G$ laparoscopic sleeve gastrectomy, \%EWL excess weight loss percentage fistulas are difficult to treat, and laparoscopic and surgical approaches have been suggested [22]. In LSG, fistula has been shown to be associated with elevated intraluminal pressure. Delayed gastric emptying and distal stenosis may play a role in fistula formation [23]. Over-the-scope clips [24] and endoscopic intra-gastric drainage (EID) [25] have been prescribed for treatment. The present study found one endoscopically treated late fistula after LSG and one surgically treated late fistula after LRYGB.

The numbers of endoscopically treated complications differed significantly between LRYGB and LSG patients, being 4.6 and $1.9 \%$, respectively $(p<0.05)$.

Known risk factors for biliary complications after bariatric surgery, including acute and chronic cholecystitis, acute pancreatitis, and choledocholithiasis, are rapid weight loss, age greater than 50, female gender, cholelithiasis at time of bariatric procedure, and RYGB operation [26]. Moon et al. compared the incidences of symptomatic gallstone formation and cholecystectomy after LRYGB and LSG operations for a mean of 2 years after the operations, finding no significant difference between the two operation types [27].

In the present study, 118 patients $(15.5 \%)$ had had a cholecystectomy performed before bariatric surgery. There was no difference between the LRYGB and LSG groups regarding the number of prior cholecystectomies $(p=0.32)$. After the bariatric operation, $23(4.2 \%)$ LRYGB patients and $2(0.9 \%)$ LSG patients had a cholecystectomy, which was performed when $5(0.9 \%)$ of the LRYGB patients and 0 of the LSG patients $(p=0.32)$ were in the acute phase. Thus, there was a significant difference between LRYGB and LSG patients in the total numbers of acute and elective cholecystectomies, at 4.2 and $0.9 \%$, respectively $(p<0.05)$. However, some patients had already been diagnosed with biliary stones before their operations. Hence, the present study also analyzed the number of complications without cholecystectomies. The LRYGB group had 31 surgically treated patients who had complications without cholecystectomies, and the LSG group had 0. This difference in the number of patients treated with complications $(p<0.001)$ was significant.

A study of 934 LRYGB and 553 LSG patients found a significant difference in the number of re-operations, including both early and late complications, and suggested that LRYGB patients are at a higher risk of re-operation [6]. In addition, a recent meta-analysis of 340 LRYGB and 345 LSG patients from randomized studies found no significant difference in late complications between the operation types but found a nonstatistically significant reduction of $36 \%$ in the relative odds of late complications for the LSG (OR, 0.64; 95\% CI, 0.28-
Table 6 Logistic multivariate regression analyze regarding age, comorbidities, sex, operation type, and weight loss regarding surgical complications without cholecystectomies

\begin{tabular}{lllll}
\hline & $p$ & ODDS & Lower (95\% CI) & Upper (95\% CI) \\
\hline Age $>$ 55 & 0.162 & 0.28 & 0.05 & 1.52 \\
Age 44-55 & 0.415 & 0.81 & 0.35 & 1.86 \\
Age $<$ 45 & & 1.00 & & \\
Sex(female) & 0.805 & 0.90 & 0.38 & 2.12 \\
Hypertension & 0.081 & 0.48 & 0.21 & 2.09 \\
Diabetes & 0.824 & 0.91 & 0.40 & 0.71 \\
LSG & 0.027 & 0.05 & 0.00 & 1.04 \\
Initial EWL\% 12 months & 0.012 & 1.02 & 1.01 & \\
\hline
\end{tabular}

The excess weight loss percentage (\%EWL) is calculated of ideal body weight to a BMI of $25 \mathrm{~kg} / \mathrm{m} 2$. Penalized Firth's regression

$L S G$ laparoscopic sleeve gastrectomy, \%EWL excess weight loss percentage 
Table 7 Logistic univariate regression analyze regarding age, sex, previous operations, operation type and weight loss regarding surgical complications

\begin{tabular}{lllll}
\hline & $p$ & ODDS & Lower (95\% CI) & Upper (95\%CI) \\
\hline Sex(female) & 0.29 & 1.42 & 0.74 & 2.7 \\
Age > 55 & 0.05 & 0.42 & 0.18 & 1.00 \\
Age 44-55 & 0.32 & 0.73 & 0.40 & 1.35 \\
Age < 45 & & 1.00 & & \\
BMI > 55 & 0.22 & 0.51 & 0.17 & 1.51 \\
BMI 45-55 & 0.66 & 1.14 & 0.64 & 2.05 \\
BMI > =45 & & 1.00 & 0.00 & 0.00 \\
Prior CC & 0.106 & 0.42 & 0.15 & 1.20 \\
Prior GB & 1.000 & 0.00 & 0.00 & 0.00 \\
Prior other operation & 0.044 & 2.29 & 1.02 & 5.11 \\
Hypertension & 0.029 & 0.53 & 0.30 & 0.94 \\
Diabetes & 0.289 & 0.74 & 0.42 & 1.30 \\
LSG & 0.001 & 0.09 & 0.02 & 0.38 \\
EWL\%2 years & 0.003 & 1.02 & 1.01 & 1.04 \\
EWLkg 2 years & 0.004 & 1.03 & 1.01 & 1.05 \\
EWL\%1 years & 0.001 & 1.02 & 1.01 & 1.04 \\
EWLkg 1 years & 0.006 & 1.03 & 1.01 & 1.05 \\
\hline
\end{tabular}

The excess weight loss percentage (\%EWL) is calculated of ideal body weight to a BMI of $25 \mathrm{~kg} / \mathrm{m} 2$

$L S G$ laparoscopic sleeve gastrectomy $\% E W L$ excess weight loss percentage, EWLkg 1/2 years excess weight loss in 1 and 2 years' time in kilograms, Prior $C C$ prior cholecystectomy, Prior GB prior gastric banding, Prior other operation included all surgical operations in the abdomen like ventral hernia and fundoplication

$1.47 ; p=0.3$ ) [28]. The present study found significantly fewer late complications that were surgically or endoscopically treated in the LSG group $(p<0.05)$. The reason for this may be as follows: LSG is a technically less-demanding operation that involves less tissue manipulation and avoids creating the anatomical anastomosis and mesenteric traps that can result in IHs or problems related to changed anatomy. In the present study, the LSG patients were older and heavier, but, in the adjusted regression model, age and pre-operative weight did not appear to be risk factors. Instead, LSG was a protective factor for surgically treated complications.

It has been stated that rapid weight loss increases the risk of IHs [29] and biliary complications [26]. In the present study's logistic multivariate regression model, 12 months weight loss was a risk factor for surgical complications. In the study, the LSG patients were heavier before their operations, and their weight reduction was smaller at both the 1- and 2-year marks than were those of the LRYGB patients $(p<0.05)$. However, previous studies have reported that a higher baseline BMI was a significant predictor of less weight loss [30]. Because of the difference in pre-operative weights between the groups in the present study, weight reduction attributed to LRYGB and LSG could not reliably be compared.

The present study controlled for weight reduction at 1 and 2 years after the operations, but, 2 years after the operations, data were missing from $41 \%$ of patients' weight records. This is because in the later years of the period, studied patients were being transferred to routine follow-ups in their own healthcare centers by 1 year after their operations. One-year weight reduction was used in the logistic regression model, because it had a follow-up rate of $75.9 \%$.

The follow-up period after the bariatric operations was 24 months, and, as mentioned, some complications can develop after this time period [12]. Hence, a longer follow-up time would have provided information on the rates of very late complications. However, the mean time for first surgical intervention was at 390 days for LRYGB patients and 388 days for LSG patients $(p=0.98)$. Therefore, the fact that late complications occurred closer to 1 year after surgery than to 2 years after suggests that a longer time period for the study was not needed.

Among the weaknesses of the present study are its retrospective nature and that the data were collected from a single hospital. In addition, it is possible that not all the complications were registered, because some of the patients were from different hospital districts, and general surgical problems, including cholecystitis and incisional hernia, may have been treated in different hospitals. However, surgical problems related directly to bariatric operations, such as $\mathrm{IH}$, were most likely referred to the hospital involved in the study. In addition, if some complications were missing from the data, similar losses could be expected in both the LRYGB and LSG groups.

Therefore, despite its limitations, the present study provides data on late complications after bariatric surgery and can aid in creating guidelines for choosing a primary bariatric procedure. If larger databases confirm the clear difference in late complications between the two operation types, without significant differences in results, the trend toward LSG noted here is justified.

\section{Conclusions}

Our data strongly support SG as the procedure of choice in bariatric surgery when it is suitable for the patient.

\section{Compliance with Ethical Standards}

Conflict of Interest The authors declare that they have no conflict of interest or any disclosure to mention.

Informed Consent Informed consent was obtained from all individual participants included in the study. 


\section{References}

1. Walsh C, Karmali S. Endoscopic management of bariatric complications: a review and update. World J Gastrointest Endosc.7(5).

2. Mokdad AH, Marks JS, Stroup DF, Gerberding JL. Correction: actual causes of death in the United States, 2000. JAMA. 293(3).

3. Angrisani L, Santonicola A, Iovino P, Formisano G, Buchwald H, Scopinaro N. Bariatric Surgery Worldwide 2013. Obes Surg. 25(10).

4. Kruger RS, Pricolo VE, Streeter TT, Colacchio DA, Andrade UA. A bariatric surgery center of excellence: operative trends and longterm outcomes. J Am Coll Surg. 218(6).

5. Colquitt JL, Pickett K, Loveman E, Frampton GK. Surgery for weight loss in adults. Cochrane Database Syst Rev. (8):CD003641.

6. Zak Y, Petrusa E, Gee DW. Laparoscopic Roux-en-Y gastric bypass patients have an increased lifetime risk of repeat operations when compared to laparoscopic sleeve gastrectomy patients. Surg Endosc. 30(5).

7. Young MT, Gebhart A, Phelan MJ, Nguyen NT. Use and outcomes of laparoscopic sleeve gastrectomy vs laparoscopic gastric bypass: analysis of the American College of Surgeons NSQIP. J Am Coll Surg. 220(5).

8. Fried M, Yumuk V, Oppert JM, Scopinaro N, Torres AJ, Weiner R, Yashkov Y, Fruhbeck $G$ et al. Interdisciplinary European Guidelines on metabolic and bariatric surgery. Obes Facts. 6(5).

9. Helmio M, Victorzon M, Ovaska J, Leivonen M, Juuti A, Jaser N, Peromaa P, Tolonen P, Hurme S, Salminen P. SLEEVEPASS: a randomized prospective multicenter study comparing laparoscopic sleeve gastrectomy and gastric bypass in the treatment of morbid obesity: preliminary results. Surg Endosc. 26(9).

10. Al-Mansour MR, Mundy R, Canoy JM, Dulaimy K, Kuhn JN, Romanelli J. Internal hernia after laparoscopic antecolic Roux-enY gastric bypass. Obes Surg. 25(11).

11. Geubbels N, Lijftogt N, Fiocco M, van Leersum NJ, Wouters MW, de Brauw LM. Meta-analysis of internal herniation after gastric bypass surgery. Br J Surg. 102(5).

12. Paroz A, Calmes JM, Giusti V, Suter M. Internal hernia after laparoscopic Roux-en-Y gastric bypass for morbid obesity: a continuous challenge in bariatric surgery. Obes Surg. 16(11).

13. Fischer JP, Basta MN, Mirzabeigi MN, Bauder AR, Fox JP, Drebin JA, Serletti JM, Kovach SJ. A risk model and cost analysis of incisional hernia after elective, abdominal surgery based upon 12 , 373 cases: the case for targeted prophylactic intervention. Ann Surg. 263(5)

14. Basta MN, Mirzabeigi MN, Shubinets V, Kelz RR, Williams NN, Fischer JP. Predicting incisional hernia after bariatric surgery: a risk stratification model based upon 2161 operations. Surg Obes Relat Dis. 12(8).

15. Coblijn UK, de Raaff CA, van Wagensveld BA, van Tets WF, de Castro SM. Trocar port hernias after bariatric surgery. Obes Surg. 26(3).
16. Pitt T, Brethauer S, Sherman V, Udomsawaengsup S, Metz M, Chikunguwo S, Chand B, Schauer P. Diagnostic laparoscopy for chronic abdominal pain after gastric bypass. Surg Obes Relat Dis. $4(3)$.

17. Greenstein AJ, O'Rourke RW. Abdominal pain after gastric bypass: suspects and solutions. Am J Surg. 201(6).

18. Ramaswamy A, Lin E, Ramshaw BJ, Smith CD. Early effects of Helicobacter pylori infection in patients undergoing bariatric surgery. Arch Surg. 139(10).

19. Azagury DE, Abu Dayyeh BK, Greenwalt IT, Thompson CC. Marginal ulceration after Roux-en-Y gastric bypass surgery: characteristics, risk factors, treatment, and outcomes. Endoscopy. 43(11).

20. Joo MK. Endoscopic approach for major complications of bariatric surgery. Clin Endosc. 50(1).

21. Eisendrath P, Deviere J. Major complications of bariatric surgery: endoscopy as first-line treatment. Nat Rev Gastroenterol Hepatol. 12(12).

22. Filho AJ, Kondo W, Nassif LS, Garcia MJ, Tirapelle Rde A, Dotti CM. Gastrogastric fistula: a possible complication of Roux-en-Y gastric bypass. JSLS. 10(3).

23. Yehoshua RT, Eidelman LA, Stein M, Fichman S, Mazor A, Chen J, Bernstine H, Singer P, Dickman R, Beglaibter N, Shikora SA, Rosenthal RJ, Rubin M. Laparoscopic sleeve gastrectomy-volume and pressure assessment. Obes Surg. 2008;18(9):1083-8

24. Kothari TH, Haber G, Sonpal N, Karanth N. The over-the-scope clip system - a novel technique for gastrocutaneous fistula closure: the first North American experience. Can J Gastroenterol. 26(4).

25. Donatelli G, Dumont JL, Cereatti F, Ferretti S, Vergeau BM, Tuszynski T, Pourcher G, Tranchart H, Mariani P, Meduri A, Catheline JM, Dagher I, Fiocca F, Marmuse JP, Meduri B. Treatment of leaks following sleeve gastrectomy by endoscopic internal drainage (EID). Obes Surg. 25(7).

26. Chang J, Corcelles R, Boules M, Jamal MH, Schauer PR, Kroh MD. Predictive factors of biliary complications after bariatric surgery. Surg Obes Relat Dis. 12(9).

27. Moon RC, Teixeira AF, DuCoin C, Varnadore S, Jawad MA. Comparison of cholecystectomy cases after Roux-en-Y gastric bypass, sleeve gastrectomy, and gastric banding. Surg Obes Relat Dis. 10(1).

28. Osland E, Yunus RM, Khan S, Memon B, Memon MA. Late postoperative complications in laparoscopic sleeve gastrectomy (LVSG) versus laparoscopic Roux-en-y gastric bypass (LRYGB): meta-analysis and systematic review. Surg Laparosc Endosc Percutan Tech. 26(3).

29. Ochner CN, Jochner MC, Caruso EA, Teixeira J, Xavier Pi-Sunyer F. Effect of preoperative body mass index on weight loss after obesity surgery. Surg Obes Relat Dis. 9(3).

30. Schneider C, Cobb W, Scott J, Carbonell A, Myers K, Bour E. Rapid excess weight loss following laparoscopic gastric bypass leads to increased risk of internal hernia. Surg Endosc. 25(5). 